\title{
REFLEXIONES SOBRE LA INTERVENCIÓN DEL TRABAJADOR SOCIAL EN EL CONTEXTO EDUCATIVO
}

\section{ELENA ROSELLÓ NADAL}

Profesora Ayudante del Departamento de Trabajo Social y Servicios Sociales. Universidad de Alicante.

\section{RESUMEN}

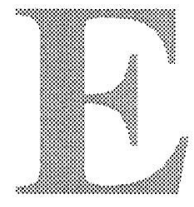

1 artículo que voy a desarrollar a continuación quiere difundir la labor que están realizando un grupo de profesionales, $y$, en concreto, de trabajadores sociales, en un ámbito de intervención poco conocido como es el contexto educativo.

En el ámbito educativo hay una importante confusión en cuanto a las funciones que realiza el trabajador social como miembro de los equipos interdisciplinares que actúan en los centros escolares, también se carece de una definición normativa que aclare nuestra situación. Y, como consecuencia de esto, la figura del trabajador social en el campo educativo tiene muy poco reconocimiento.

El artículo recoge las reflexiones que he realizado en mis años de ejercicio profesional como trabajadora social en los Servicios Psicopedagógicos Escolares (S.P.E.) de la Consellería de Educación y Ciencia, así como las aportaciones realizadas por otros compañeros que trabajan en este ámbito.

Por eso, creo importante aportar un poco de claridad en algunos aspectos: contextualizar la figura del trabajador social dentro del marco educativo y, en concreto, dentro de los equipos interdisciplinares que actúan en los centros escolares; intentar delimitar cuáles serían las funciones específicas que podrían desarrollar los trabajadores sociales; reflexionar sobre la metodología de trabajo que debería utilizarse para que nuestra intervención fuera realmente eficaz y eficiente y presentar algunas propuestas de intervención a nivel operativo que podrían darse en la práctica cotidiana.

Considero fundamental la divulgación de materiales, documentos, programas e investigaciones con los que se esté trabajando en la práctica profesional para contribuir al afianzamiento de la disciplina del 
Trabajo Social en el ámbito educativo y para dejar abierto un campo profesional en el que considero necesario e imprescindible la figura del trabajador social.

\section{PALABRAS CLAVE}

Trabajo social escolar; orientación educativa y sociolaboral; diversidad del alumnado; valoración sociopsicopedagógica; asesoramiento a padres; modelo ecológico-sistémico.

\section{INTRODUCCIÓN}

El sistema educativo no ha sido capaz de absorber y modificar toda la problemática que el alumnado lleva de forma individual y que está dificultando la consecución de los objetivos que la institución escolar se plantea para con los alumnos.

Si concebimos el proceso educativo como un todo en el que se pretende conseguir que el alumno tenga una situación tal que posibilite su desarrollo libre y armónico, tendremos en cuenta que son necesarias acciones, no sólo por parte de los educadores, sino también por parte de otros profesionales (psicólogos, pedagogos, trabajadores sociales, logopedas, etc.). Estos profesionales tienen su razón de ser porque vienen a cubrir lagunas existentes en el propio sistema educativo, tanto en el desarrollo en sí, mediante la superación de las dificultades individuales y colectivas que encierran, como en su acción preventiva.

La actividad del trabajador social no es sino un eslabón en la cadena; su grado de eficacia será mayor cuando más se realice dentro del trabajo y actividades del equipo educativo.

El modelo de intervención en un centro debe tener en cuenta el carácter sistemático del contexto en el que se desarrolla el acto educativo. Somos conscientes de que para que "los árboles no nos impidan ver el bosque", nuestra perspectiva tiene que dejar de centrarse en los alumnos o en las situaciones problema, considerándolas de forma aislada.

Este punto de vista reduccionista sólo nos permite salir del paso momentáneamente, suprimir unos síntomas, mostrar una respuesta a una demanda poco clarificada. Pero a la larga nos crea un sentimiento de insatisfacción o frustración por su exigua operatividad. Debemos entender el acto educativo como una serie de procesos que suceden inscritos en un sistema más amplio, sobre él hay que actuar de forma 
global, o al menos teniendo en cuenta las posibles interacciones de las partes. Este cambio de perspectiva pasa necesariamente por establecer un puente o una unión entre educadores y profesionales de los equipos de apoyo a los centros, que permita contextualizar las situaciones en el marco global en el que se encuentran. Y, al mismo tiempo, conlleva una actitud de evaluación crítica y de investigación sobre nuestro propio trabajo, modificando el mismo en función de su operatividad.

En este artículo se pretende difundir un trabajo "silencioso" que vienen desarrollando un grupo de profesionales, trabajadores sociales, dentro del contexto educativo y, digo silencioso, porque, a pesar de los años que llevan ejerciendo su profesión dentro de este contexto (más de 15 años desde los primeros pioneros) y del inestimable valor de sus aportaciones profesionales (aspecto éste que intentaré demostrar a lo largo de este artículo), siguen siendo unos profesionales muy poco conocidos dentro de este contexto y apenas imperceptibles si nos referimos a ámbitos ajenos a donde estos trabajadores sociales realizan su labor, siendo, en general, una profesión poco conocida por la sociedad.

\section{FUNDAMENTACIÓN TEÓRICA}

La Federación Internacional de Trabajadores Sociales (F.I.T.S.) afirma que el medio escolar es el primero en el que se pueden detectar problemas familiares y sociales. Considera a la institución escolar como uno de los pilares de prevención, ya que en ella se pueden detectar posibles anomalías antes que en otras instituciones, de forma globalizada, y que facilitaría una intervención temprana para modificar, en la medida de lo posible, la situación que está influyendo negativamente.

En el contexto educativo, el niño está integrado básicamente en tres subsistemas: la escuela, el grupo-clase y la familia. Dentro de cada uno de ellos, establece una serie de relaciones; estas relaciones y las interacciones que mantienen los diferentes sistemas entre sí determinarán el papel que el niño desempeñe tanto en la escuela como en casa; de ahí que nuestra intervención profesional, cuyo objetivo último es favorecer el desarrollo integral del alumno, tenga que incidir en los diferentes contextos en los que el niño se desenvuelve.

Si el medio familiar y comunitario en el que se desenvuelve el niño es un medio "normalizado" y el centro escolar responde a las expectativas e intereses del niño, entonces no surgirán problemas, pero en el momento en que alguna de estas áreas sea deficitaria o cuando las relaciones que se establecen entre ellas sea disfuncional seguramente será 
justificada nuestra intervención como trabajadores sociales para intentar corregir los desajustes personales, familiares, sociales y/o escolares que se manifiesten en el niño.

Toda situación educativa afecta al niño en su unidad como persona: lo biológico, lo afectivo, lo intelectual y lo social forman parte del niño en una realidad interactiva, de modo que no cabe aislar en comportamientos separados cada una de las dimensiones constitutivas de la personalidad.

El Trabajo Social en el ámbito educativo y dentro de un equipo interdisciplinar, como es el Servicio de Orientación Educativa, Psicopedagógica y Profesional, tiene su razón de ser en la necesidad de abordar la realidad en la que se interviene desde una perspectiva globalizadora, que tenga en cuenta todos los factores y elementos que interactúan en el proceso educativo, tanto en el sistema escolar como en su relación con otros sistemas e instituciones.

Podría definirse el Trabajo Social Escolar como la ayuda técnica que favorece el establecimiento y fortalecimiento de las relaciones entre el medio escolar, el familiar y el comunitario, la integración escolar y social de los niños que tienen dificultades de adaptación al contexto educativo por sus circunstancias personales, familiares o sociales y la intervención sobre todos aquellos obstáculos que impiden el desarrollo integral del menor.

En la adaptación al medio escolar del alumno influyen enormemente factores vinculados a la familia, como el clima cultural, las motivaciones hacia el estudio, la valoración del trabajo escolar, el clima afectivo y el apoyo y seguimiento de los progresos y esfuerzos del niño.

Numerosos estudios han demostrado que la incidencia negativa de los factores ambientales se detecta en mayor proporción en las familias de ambientes socioculturales desfavorecidos, pero no de forma exclusiva; cada vez más, en las familias que podríamos considerar normalizadas, se aprecia la carencia de elementos básicos para el desarrollo adecuado del niño, como la motivación y la ayuda para las tareas escolares, un clima afectivo apropiado, unas normas y valores claramente establecidos y respetados, unos mecanismos de refuerzo y recompensa ajustados, etc.

Así pues, la intervención del trabajador social en este ámbito no puede tener otro objetivo que el de contribuir a que el alumno, independientemente de su pertenencia a un entorno sociofamiliar u otro, tenga las mismas oportunidades educativas que los demás, procurando que el entorno más inmediato del alumno sea lo más motivador y estimulante posible, de cara a su adaptación y rendimiento escolar, trabajando conjuntamente con las familias e implicándolas al máximo en el proceso educativo. 
Los trabajadores sociales realizarán su intervención socioeducativa en y con la comunidad educativa, y para el desarrollo de su trabajo estarán en constante coordinación con tutores, familias, centros y profesionales de otros servicios con el fin de garantizar el máximo aprovechamiento de los recursos sociales disponibles.

\section{FUNDAMENTACIÓN LEGISLATIVA}

La legislación básica internacional sobre la que se basa la atención a las necesidades socioeducativas de los niños es la Convención sobre los Derechos del Niño. Este documento reconoce el derecho del niño a la educación, determinando que los estados firmantes, a fin de que se pueda ejercer progresivamente y en condiciones de igualdad de oportunidades ese derecho, deberán en particular:

"Hacer que todos los niños dispongan de información y orientación en cuestiones educacionales y tengan acceso a ellas». «Adoptar medidas para fomentar la asistencia regular a las escuelas y reducir las tasas de deserción escolar».

Por otro lado, la actuación de los trabajadores sociales en el ámbito escolar queda contextualizada en la Ley Orgánica 1/1990, de 3 de octubre de Ordenación General del Sistema Educativo (a la que nos referiremos a partir de ahora como LOGSE), en el preámbulo de dicha ley se señala "el objetivo primero y fundamental de la educación es el de proporcionar a los niños y niñas, a los jóvenes de uno y otro sexo una formación plena que les permita conformar su propia y esencial identidad... Tal formación plena ha de ir dirigida al desarrollo de su capacidad para ejercer, de manera crítica y en una sociedad axiológicamente plural, la libertad, la tolerancia y la solidaridad."

En el Título Preliminar, artículo 2, se señalan los principios que guiarán la actividad educativa y, entre ellos, señalo, como más significativos, los siguientes:

c) la efectiva igualdad de derechos entre los sexos y el rechazo a todo tipo de discriminación, y el respeto a todas las culturas,

d) la atención psicopedagógica y la orientación educativa y profesional,

j) la relación con el entorno social, económico y cultural

Cuando habla de la Educación Especial (Título primero, Capítulo quinto) en el artículo 36, punto 2, dispone que "la identificación y valoración de las necesidades educativas especiales se realizará por equipos integrados por profesionales de distintas cualificaciones, que establecerán en cada caso planes de actuación en relación con las necesidades educativas específicas de los alumnos". 
En el Título Cuarto, referido a la Calidad de la Enseñanza, se recoge, en el artículo 60, punto 2, lo siguiente: "Las Administraciones educativas garantizarán la orientación académica, psicopedagógica y profesional de los alumnos, especialmente en lo que se refiere a las distintas opciones educativas y a la transición del sistema educativo al mundo laboral, prestando singular atención a la superación de hábitos sociales discriminatorios que condicionan el acceso a los diferentes estudios y profesiones. La coordinación de las actividades de orientación se llevará a cabo por profesionales con la debida preparación."

Hay que remarcar, dentro de esta ley, el Título quinto, de la Compensación de las Desigualdades en la Educación, que, en su artículo 63, establece que "las políticas de educación compensatoria reforzarán la acción del sistema educativo de forma que se eviten las desigualdades derivadas de factores sociales, económicos, culturales, geográficos, étnicos o de otra indole" y, posteriormente (artículo 64), añade: "Las Administraciones educativas asegurarán una actuación preventiva y compensatoria garantizando, en su caso, las condiciones más favorables para la escolarización, durante la educación infantil, de todos los niños cuyas condiciones personales, por la procedencia de un medio familiar de bajo nivel de renta, por su origen geográfico o por cualquier otra circunstancia, supongan una desigualdad inicial para acceder a la educación obligatoria y para progresar en los niveles posteriores."

Con el fin, pues, de asegurar la necesaria calidad de la enseñanza, la citada ley ordena en su disposición adicional tercera, 3 e), que "en el proceso de su aplicación las administraciones educativas crearán servicios especializados de orientación educativa, psicopedagógica y profesional que atiendan a los centros que impartan enseñanzas de régimen general."

Habiendo hecho ya un análisis exhaustivo del marco legal genérico que supone la LOGSE para la actuación de los equipos multiprofesionales en el ámbito educativo, pasaré ahora a hacer una revisión de la normativa autonómica más concreta que regula la creación y funcionamiento de estos equipos dentro del ámbito de nuestra Comunidad Valenciana.

El Decreto 136/1984, de 10 de diciembre, del Gobierno Valenciano, creó los Servicios Psicopedagógicos Escolares (S.P.E.), integrando en éstos a los equipos multiprofesionales establecidos por la Ley 13/ 1982, de 7 de abril, de Integración Social de los Minusválidos (L.I.S.M.I.) y definiendo un modelo de intervención propio de la Comunidad Valenciana. 
Posteriormente, el Decreto 53/1989, de 18 de abril, del Gobierno Valenciano, definió un nuevo marco jurídico de los servicios psicopedagógicos escolares y de los gabinetes psicopedagógicos municipales. Por otra parte, el Real Decreto 986/1991, de 14 de junio, por el que se aprueba el calendario de aplicación de la nueva ordenación del sistema educativo, en su artículo 18, establece que "las administraciones educativas procederán a la creación progresiva de servicios especializados de orientación educativa, psicopedagógica y profesional para atender a los centros docentes de manera que el proceso quede completado en el momento de la implantación total de los respectivos niveles y etapas del nuevo sistema."

Y, así, el Decreto 13/1994, de 5 de julio, del Gobierno Valenciano, regula los servicios especializados de orientación educativa, psicopedagógica y profesional de la Conselleria de Educación y Ciencia y los define como "equipos integrados por distintos profesionales que se constituyen para favorecer el ejercicio de funciones especializadas de orientación, evaluación e intervención educativa y, en general, de apoyo al sistema escolar en sus distintos niveles educativos y su vinculación al mundo del trabajo."

\section{FUNCIONES GENERALES}

El Decreto 131/1994 establece, como funciones generales de los servicios especializados de orientación educativa, psicopedagógica y profesional, las siguientes:

a) Participar en el apoyo y asesoramiento a los centros educativos.

b) Elaborar y difundir materiales e instrumentos de orientación educativa y de intervención sociopsicopedagógica y logopédica en las dos lenguas oficiales de la Comunidad Valenciana.

c) Coordinar las actividades de orientación educativa y sociofamiliar que se realicen en los centros docentes de su ámbito de actuación.

d) Asesorar al profesorado en el tratamiento de la diversidad del alumnado.

e) Asesorar al profesorado en el diseño de procedimientos e instrumentos de evaluación, tanto de los aprendizajes del alumnado, como del mismo proceso de enseñanza.

f) Detectar aquellas condiciones personales y sociales que faciliten o dificulten el proceso de enseñanza y aprendizaje del alumnado y su adaptación al ámbito escolar.

g) Realizar la evaluación y la valoración sociopsicopedagógica y logopédica del alumnado, para la determinación de su escolarización más adecuada y, si procede, elaborar la propuesta de diversifica- 
ción curricular o de adaptación curricular significativa y, en su caso, realizar el tratamiento logopédico y rehabilitador.

h) Colaborar en la orientación académica, para favorecer en el alumnado la toma de decisiones.

i) Colaborar en la orientación del alumnado en los procesos de transición a otras etapas e itinerarios educativos y al mundo del trabajo.

j) Llevar a cabo la orientación psicopedagógica sobre el proceso de enseñanza y aprendizaje y sobre la adaptación personal y social en el ámbito educativo.

k) Asesorar a las familias o a los representantes legales del alumnado, participando, en su caso, en el desarrollo de programas formativos de padres y madres de alumnos.

l) Cualquier otra que se determine reglamentariamente.

El artículo quinto, punto 4, referido a la organización, recoge un aspecto muy importante que es el de la coordinación con los profesionales de los recursos y servicios sociocomunitarios, en la línea de elaborar acciones conjuntas adecuadas a las demandas planteadas, con el objeto de proporcionar respuestas globalizadas.

En el artículo sexto, punto 2, del citado Decreto, referido a la composición de estos equipos, establece que "los profesionales que compondrán los servicios psicopedagógicos escolares podrán ser... C) Trabajadores sociales".

\section{FUNCIONES PRIORITARIAS}

La Orden de 10 de marzo de 1995 por la que se determinan las funciones de cada profesional dentro de los equipos, señala como funciones prioritarias del trabajador social las siguientes:

a) Asesorar a la comunidad educativa sobre aspectos familiares y sociales del alumnado y sobre los recursos socioeducativos de la zona.

b) Realizar el estudio y la valoración sociofamiliar de los casos detectados, bien elaborando la propuesta de intervención socioeducativa o bien derivándolo hacia el recurso pertinente, con el posterior seguimiento y evaluación del caso.

c) Participar en el desarrollo de programas de garantía social, de orientación escolar o profesional y en programas formativos para padres y madres de alumnos.

Partiendo de esta determinación normativa y desarrollándola, voy a intentar señalar las funciones, actividades y programas que podrían desarrollar los trabajadores sociales en el ámbito educativo, intentando abrir un amplio abanico de posibilidades, que en cada realidad con- 
creta deberán ser valorados y priorizados adecuadamente, atendiendo al contexto particular en el que se interviene.

Las condiciones y elementos de trabajo con los que se encuentre cada profesional determinarán el énfasis en unas líneas de actuación u otras, el carácter de la intervención y el nivel de implicación en las tareas, asumiendo una mayor o menor responsabilidad en su realización.

\section{FUNCIONES ESPECÍFICAS DEL TRABAJADOR SOCIAL}

Sus funciones específicas serán las siguientes:

a) Participar en la elaboración y desarrollo de programas de prevención y detección dirigidos a la comunidad educativa.

b) Asesorar al profesorado en la elaboración, seguimiento y evaluación de los programas de acción tutorial (P.A.T.) y en la atención a la diversidad.

c) Realizar el estudio y la valoración sociofamiliar de los casos detectados. Elaborar la propuesta de intervención socioeducativa directa o canalizar hacia el recurso pertinente, con el posterior seguimiento y evaluación del caso.

d) Participar en la elaboración y realización de actividades de orientación educativa y sociolaboral.

e) Colaborar con los distintos órganos de representación del centro, según necesidades.

f) Asesorar a las familias y participar, en su caso, en el desarrollo de programas formativos de padres y madres de alumnos.

g) Elaborar documentos y materiales propios de la intervención socioeducativa.

\section{ALGUNAS CONSIDERACIONES PREVIAS A LA INTER- VENCIÓN}

Antes de pasar a describir las actividades y programas que pueden desarrollar los trabajadores sociales en este ámbito, quisiera presentar unas reflexiones que considero deberíamos hacernos previamente a nuestra intervención.

\subsection{Modelo ecológico-sistémico}

Personalmente parto de la idea de que debe haber una transformación (que yo denominaría evolución) de lo que tradicionalmente ha 
sido la intervención habitual de los trabajadores sociales en este campo. Con esto, me refiero a que si hasta ahora el nivel de intervención que se ha venido utilizando ha sido el nivel individual $y$, en algunos aspectos, el familiar, es necesario ir introduciendo también el nivel de intervención comunitario y, más que eso, superando esta tradicional fragmentación de nuestra intervención en diferentes niveles apuesto por un modelo de intervención integral e integrado, que asuma el enfoque de la intervención desde un modelo teórico-metodológico como el ecológico-sistémico.

Este modelo puede considerar al menor como miembro de una familia, que, a su vez, está inmersa en su entorno, con el cual establece una serie de relaciones interpersonales e intergrupales, en donde ambos sistemas (familiar- comunitario) están interconectados constantemente; pero, a su vez, también puede considerar al menor como un alumno dentro de otro sistema, el escolar, y las interrelaciones que se establecen entre ese sistema escolar y el sistema familiar.

Este enfoque de la intervención resulta difícil por varias razones:

- Es necesaria la clarificación de roles para no "perder los papeles" en esa intervención conjunta de profesionales de diferentes disciplinas. Cada profesional tiene que tener claramente diferenciada su área competencial, dejándola explícita desde un principio, de modo que no conlleve dificultades de entendimiento, pero tampoco se interprete como un distanciamiento o una barrera para no querer trabajar en equipo.

- Hay una necesidad de formación de carácter metodológico en algunas profesiones que posibilite el crear una infraestructura de intervención que permita el trabajo en equipo y actúe contextualizando las situaciones, es decir, una metodología que potencie el enriquecimiento y la aportación mutua de diferentes disciplinas y ayude a ver más allá de lo evidente.

- Los problemas del sistema educativo están interrelacionados. Sin embargo, conviene delimitar qué situaciones están bajo el efecto de nuestra intervención y cuáles no. A veces, una postura cómoda es culpar a agentes externos sobre los que no tenemos capacidad ni responsabilidad de intervención. Con ello, justificamos la inoperancia de nuestro trabajo cuando el cambio nos supone un gran esfuerzo o, simplemente, cuando no somos capaces de asumir nuestra parte de responsabilidades de la situación, ante lo cual nos sentimos angustiados e impotentes.

- La Administración Educativa (en nuestro caso, la Consellería de Educación y Ciencia) asigna una determinada zona de intervención a cada S.P.E., y, así, cada equipo se ve obligado, por distintas razones, a intervenir en muchos centros; además, a esto se añade que 
normalmente hay un solo trabajador social en cada equipo con lo cual la ratio de centros y demandas a atender por el trabajador social se ve ampliamente sobrepasada. Esto provocará que en muchas situaciones sólo se pueda intervenir sobre demandas superficiales (aquellas referidas a los síntomas, los aspectos individuales y que no se cuestionan el contexto en el que se sitúan los problemas), lo que a la larga resulta poco útil y operativo, y, lo que es peor, desmotivador para el profesional.

\subsection{Modelo de investigación-acción}

La investigación-acción es un método de trabajo por el que un grupo de profesionales, conscientes de unas necesidades, ponen en práctica un proceso de cambio para satisfacer las mismas. La actuación profesional de los miembros del equipo es diferenciada, pero basando esta diferencia exclusivamente en sus distintos roles profesionales y en su distinta área de competencias. La deben realizar los profesionales de los S.P.E., el profesorado del centro, así como otros profesionales que puedan intervenir en la situación, en un ambiente de participación y compromiso. Esto evitará la actitud pasiva por parte del profesorado, cuando expone su demanda y espera que el S.P.E. le dé la solución y también evita, por otra parte, la actitud descomprometida de los profesionales del S.P.E. que terminan su intervención con la devolución del informe o la derivación de la demanda a otro servicio.

El proceso de investigación-acción comprendería los siguientes pasos:

1. Identificar o clarificar una relación de problemas. Es imprescindible hacer explícito un listado de situaciones-problema y realizar una jerarquización del mismo. Esta priorización de problemas debe ser revisada constantemente en el transcurso del proceso.

2. Exploración. Supone indagar unos hechos acerca de las circunstancias que concurren en la idea general susceptible de cambio. Requiere:

a) Describir del modo más completo posible la naturaleza de la situación que se desea cambiar o mejorar.

b) Explicar los hechos de la situación, analizando el contexto en el que surgen. Comprende:

- Generar hipótesis explicativas.

- Comprobación de las hipótesis, si se puede establecer alguna relación entre la situación y algún otro factor que opere en el contexto.

c) Construcción del Plan General de Acción. Debería contener:

a) Una descripción revisada de la idea general, clarificada. 
b) Descripción de los factores que se ha de modificar o cambiar y las acciones que se ha de emprender en este sentido.

c) Descripción de los recursos con los que se cuenta.

d) Descripción del compromiso establecido entre las personas que intervienen.

4. Desarrollo de los pasos siguientes del Plan. Especificar la temporalización de las medidas previstas en el Plan general y cómo han de ser verificados los procesos de puesta en marcha, su efectividad.

5. Puesta en marcha de los pasos siguientes del Plan, en el caso de que haya consecuencias sobre las que deba tratarse. Implica una revisión constante del Plan General de Acción.

\subsection{Modelo de intervención por programas}

Un tercer aspecto a considerar, y relacionado con los anteriores, es que debemos ir transformando nuestro esquema tradicional de intervención (intervención sobre casos detectados) por un modelo de intervención por programas.

Este modelo debe partir de un análisis de necesidades y demandas de la comunidad educativa y, a partir de ahí, el trabajador social, como miembro integrante de un equipo interdisciplinar, elaborará, junto con el resto del equipo y tras una negociación con cada centro escolar (a través de la Comisión de Coordinación Pedagógica de cada centro), un plan de actuación general que se estructurará en diferentes programas adaptados a cada contexto específico. Habrá que lograr implicar a un grupo de profesores que sean capaces de dinamizar un determinado programa desde el propio centro, dándoles la información y formación necesaria que les capacite para poder desarrollar las diferentes actividades del programa, pero contando siempre con el asesoramiento técnico y profesional necesario por parte de uno o varios especialistas que coordinen dichas actividades.

Esto supondría una reestructuración de la atención a los centros, una priorización de funciones y actividades en detrimento de otras, una mayor coordinación con otros profesionales para lograr una cierta unidad y coherencia en las acciones a desarrollar y renunciar a ciertos comportamientos y conceptualizaciones (paternalistas o asistencialistas) para trabajar por programas de forma progresiva y secuenciada.

Este proceso de transición hacia un modelo de actuación por programas no es nada fácil puesto que supone un cambio de actitud personal y profesional del trabajador social e implicaría, a su vez, un nuevo modelo organizativo que afectaría a los centros y al propio equipo, cambios estos para los cuales no todos pueden estar mentalizados. Este es el nuevo planteamiento que tendremos que asumir para adaptarnos a 
los principios y los cambios que plantea la LOGSE, tendremos que intentar superar las intervenciones de tipo puntual e individualizado para dar paso a una concepción más globalizadora e integradora.

Otro de los grandes retos que se le plantean al trabajador social en este campo es convencer a la institución escolar, y a la comunidad educativa, en general, de que resulta mucho más rentable (tanto cuantitativa como cualitativamente) invertir todo tipo de medios (materiales, técnicos, económicos, humanos, etc.) en actividades y programas de prevención que en intervenciones puntuales de parcheo cuando la situación está ya bastante deteriorada.

Para poder actuar desde este punto de vista más preventivo y globalizador es necesario que se den unas premisas básicas:

1. Compromiso por parte del centro de potenciar actividades secuenciadas en programas para todos los alumnos, a pesar de la falta de habituación para trabajar por programas.

2. Implicación y disponibilidad de tiempo del personal que interviene. Puesto que se ha de partir de un diagnóstico de necesidades, éstas se han de priorizar y concretar en el diseño de un programa que hay que elaborar; posteriormente, tendremos que ejecutar lo diseñado $y$, finalmente, evaluar lo ejecutado (esto supone un esfuerzo de tiempo y de preparación que ha de ser reconocido por todos).

3. Disponibilidad de recursos humanos, materiales, económicos y técnicos para llevar a cabo este plan de actuación general.

4. La unidad de intervención puede ser el grupo-clase, un determinado nivel educativo o el centro, en general, e irá dirigido a todos los alumnos, no sólo a los que presenten algún tipo de problemática específica.

Resulta obvio señalar que para que pueda darse esta intervención por programas es necesaria la colaboración de otros profesionales, organismos e instituciones en todas las fases del mismo (diagnóstico, diseño, implementación, ejecución y evaluación), así como de todos los agentes sociales y educativos implicados en la situación (profesores, padres, tutores, alumnos, profesionales, etc.).

\section{ACTIVIDADES Y PROGRAMAS QUE PUEDEN DESARRO- LLAR LOS TRABAJADORES SOCIALES EN EL ÁMBITO ESCOLAR.}

Intentar delimitar las líneas de intervención que pueden realizar los trabajadores sociales en el ámbito educativo es un objetivo un tanto utópico, y digo esto por varios motivos: 
1. Cada trabajador social realizará su intervención dentro de un contexto determinado, de una realidad concreta, que va a ir marcando y delimitando mucho su intervención profesional. Y no se puede (o no debemos) coger un programa que nos parece interesante y aplicarlo sin más a nuestro contexto concreto sin haber valorado previamente la adecuación e idoneidad del mismo a nuestra realidad concreta. Habrá que tener en cuenta una serie de aspectos tales como: necesidad real o sentida de ese programa, características de los usuarios a los que va dirigido, recursos y medios con los que se cuenta, grado de implicación del equipo o de otros profesionales, etc.

2. Cada profesional, por una serie de condicionantes propios a nivel personal, académico, formativo, institucional, etc., va a desarrollar su trabajo desde una determinada perspectiva y puede considerar que el que se quiera delimitar su actuación desde un modelo determinado o con la utilización de una determinada metodología pueda suponer para él un desafío (si lo ve como un reto personal y profesional), una imposición (si las órdenes vienen desde arriba o la institución le obliga a ello), o, incluso, como una amenaza (si no está capacitado para responder de determinada manera, puede ver amenazado su puesto de trabajo).

3. Uno de los principales rasgos que caracterizan a nuestra profesión es la constante adaptación a los cambios que se van produciendo en todos los órdenes (económico, social, legislativo, etc.). Es por esta razón que el trabajador social tiene que estar constantemente adaptándose a todas estas transformaciones para ser capaz de dar respuestas a las nuevas necesidades sociales que puedan ir surgiendo en esta sociedad dinámica que es la nuestra. Como consecuencia de esto, lo que podemos proponer en un momento determinado, como una alternativa eficaz de intervención, puede quedar desfasado en un tiempo relativamente corto o puede ya no ser el centro de interés al año siguiente. Esto supone un riesgo en el sentido de que podemos programar actuaciones para un período de tiempo determinado $y$, cuando nos vamos a sentar a evaluar lo programado (etapa del método que pocas veces realizamos) nos encontramos que es casi inoperativo porque al año siguiente ya no se va a continuar con ese programa y lo que se nos está pidiendo es que nos pongamos ya a diseñar el programa del año siguiente que puede estar relacionado o no con el anterior.

Una vez que hemos reflexionado un poco sobre este tipo de consideraciones y otras que seguro han quedado en el tintero, puedo pasar a presentar algunos programas que considero pueden resultar interesantes para aquellas personas que trabajen en este campo o para aquellos que quieran conocer algo más sobre él. 
Es importante recordar, de nuevo, que no son programas diseñados, ejecutados y evaluados única y exclusivamente por trabajadores sociales, creemos que el trabajador social debe participar en ellos como un miembro más dentro de un equipo interdisciplinar. Es el equipo en su conjunto el que tiene que planificar estos programas. Lo que sí que habrá que definir con claridad es el grado de implicación y de responsabilidad de cada uno de los miembros del equipo y debe ser esta una decisión que comparta y asuma todo el equipo para evitar problemas posteriores cuando el programa se esté ejecutando.

Los programas y actividades a realizar van a ser presentados siguiendo la clasificación establecida en el apartado anterior de funciones específicas como una manera coherente de desarrollo de estas funciones.

\subsection{Participar en la elaboración y desarrollo de programas de pre-} vención y detección dirigidos a la comunidad educativa.

El Decreto 131/1994 señala, como una de las funciones de los Servicio de Orientación Educativa, Psicopedagógica y Profesional, la de "detectar aquellas condiciones personales y sociales que faciliten o dificulten los procesos de aprendizaje del alumnado y su adaptación al ámbito escolar". Entendemos que el desarrollo de esta función tiene una finalidad preventiva, de especial relevancia en toda intervención social y, particularmente, en el ámbito de la educación.

Anteriormente, hicimos referencia a la prevención como uno de los principios que debe regir nuestra intervención, convencidos de que las actuaciones preventivas son, sin duda, de mayor eficacia y, en última instancia, de menor coste social que las medidas paliativas. Si bien, a menudo sólo se emplea el esfuerzo y el tiempo en estas últimas, por falta de medios o por carecer de perspectivas a largo plazo, tanto en la programación como en la valoración de resultados, siendo las posibilidades de éxito infinitamente menores.

En el desarrollo de esta función, el trabajador social debe desempeñar una importante labor de coordinación, tanto con el resto de los profesionales del servicio, profesores, padres y alumnos, como con otros servicios socio-comunitarios, colaborando en la prevención y detección de indicadores que puedan incidir en el proceso evolutivo y adaptativo del alumno.

\subsubsection{Estudio de la zona de actuación.}

Es imprescindible, antes de plantearse cualquier actividad preventiva, conocer la realidad concreta, las necesidades de la comunidad 
educativa, del centro al que irán dirigidas. Este conocimiento determinará tanto los contenidos, los temas a abordar, como los medios y la metodología más apropiada. De no actuar así, se corre el riesgo de aplicar actividades o programas preventivos muy bien diseñados pero que no responden a las características y las necesidades de la población a la que se dirigen.

\subsubsection{Participar en programas de prevención inespecífica en el área socioeducativa.}

Esta actividad se refiere a actuaciones preventivas que se lleven a cabo sólo en el ámbito escolar, con alumnos, padres o profesores o en coordinación con otras instituciones (del ámbito sanitario, social...) y en contextos más amplios. Y abarcaría programas de prevención del absentismo escolar, de prevención de drogodependencias o, en términos más amplios, de educación para la salud; o bien de desarrollo personal, de habilidades y actitudes, etc., siempre dependiendo de las características y necesidades del contexto y la población a la que se dirigen.

8.1.3. Colaborar en programas de detección, previa a la escolarización, de niños con necesidades educativas especiales.

Diferentes investigaciones han puesto de manifiesto que los esfuerzos educativos de mayor eficiencia son aquellos realizados antes de los tres años de edad, período de especial importancia en el desarrollo del niño.

En el Servicio de Atención Ambulatoria Previo a la Escolarización (S.A.A.P.E) ubicado en centros específicos de Educación Especial, se atienden las necesidades específicas de los casos detectados a través de la coordinación con los servicios de salud, servicios sociales, escuelas infantiles y guarderías, asociaciones, etc.; arbitrando, lo antes posible, los recursos necesarios para que se normalice su proceso de escolarización.

Por otra parte, el trabajador social aportará la información relevante sobre el contexto socio-familiar del niño al dictamen de escolarización, cuya finalidad es determinar las necesidades educativas especiales, la modalidad de escolarización adecuada y los tratamientos y apoyos específicos. Así, se podrá realizar un trabajo preventivo desde su integración en el centro. 
8.1.4. Detección inicial, en educación infantil y primer ciclo de primaria, de situaciones de riesgo social, en colaboración con el profesor-tutor.

El desarrollo de esta actividad exige la elaboración de instrumentos para la recogida de información, o completar los que ya existen (añadir nuevos ítems al cuestionario para la historia personal del alumno...) para que los tutores registren todo aquello que pueda informar de situaciones de riesgo social en el entorno socio-familiar del alumno. Y, posteriormente, la realización de entrevistas con tutores, familias y otros profesionales, para completar la información y arbitrar las medidas necesarias para introducir modificaciones y evitar el deterioro de las situaciones detectadas.

\subsubsection{Intervención inicial en las problemáticas socioeducativas que se presenten, especialmente en Educación infantil y primer ci- clo de Primaria.}

Es enormemente importante la intervención temprana de los diferentes profesionales que trabajamos en este ámbito en el momento en que aparece algún problema en la vida escolar del alumno (absentismo, dificultades de relación con profesores o compañeros, etc.) a fin de poder atajar las causas que lo ocasionan y evitar que esa situación continúe a lo largo de la escolaridad.

\subsubsection{Establecer o potenciar mecanismos de coordinación y trasvase de información formales, con los diferentes servicios de la zona.}

Esto permitirá que, frente a situaciones o problemáticas detectadas en cualquier ámbito de intervención, sea posible una respuesta rápida y coordinada y la previsión de recursos en el área educativa o en cualquier otra.

A este respecto, son necesarios programas de coordinación interinstitucional de cara a la prevención e intervención rápida en problemáticas del menor, con el fin de optimizar la actuación de los diferentes servicios e incrementar su eficacia preventiva, con un sistema de registro de información, seguimiento y evaluación común. 
8.2. Asesorar al profesorado en la elaboración, seguimiento y evaluación de los programas de acción tutorial (P.A.T.) y en la atención a la diversidad.

Si en todas las funciones el trabajo en equipo es fundamental, aquí es cuando cobra su mayor sentido, ya que ningún profesional de la escuela (ni de ningún otro campo) puede tener conocimientos ni habilidades suficientes para dar respuesta adecuada a dos tareas que son dos de los pilares fundamentales de la educación actual. El trabajador social, como profesional que forma parte de un equipo, tiene una aportación específica, pero su trabajo, al igual que el de los demás profesionales, no tiene sentido si se realiza en solitario, ya que debe coordinarse siempre estrechamente tanto con los demás profesionales del equipo como con los profesores de los centros.

\subsubsection{Colaborar en el programa de acción tutorial.}

Las actividades que puede llevar a cabo el trabajador social en los tres niveles en los que se desarrolla la acción son las siguientes:

a) A nivel del profesorado:

- Colaborar en la elaboración, seguimiento y evaluación del PAT a nivel del centro.

- Apoyar al tutor en el desarrollo del PAT, según sus necesidades.

- Promover y facilitar la coordinación de todos los profesores que intervienen en una misma aula para la realización de las actividades del PAT.

- Favorecer la disposición hacia el intercambio de ideas y experiencias y hacia la ayuda y la colaboración.

- Potenciar actividades encaminadas a la actualización y renovación profesional.

b) A nivel del alumnado:

- Aconsejar al tutor para su mejor conocimiento del alumnado, tanto a nivel individual como de grupo.

- Guiar al tutor en la creación de un clima social positivo dentro del grupo-clase.

- Orientar al tutor en su labor de fomento de actitudes participativas en los alumnos.

- Asistir al tutor en el desarrollo de determinados valores, actitudes y habilidades en el alumnado, encaminados a favorecer su realización personal y social.

- Colaborar en el proceso de orientación (se desarrolla en el apartado referido a las actividades de orientación educativa y sociolaboral). 
c) A nivel de la familia:

- Proponer actividades al tutor para el mejor conocimiento de las familias, informándole de los aspectos relevantes del entorno sociofamiliar de sus alumnos y de cómo influye éste en el proceso de enseñanza-aprendizaje.

- Aconsejar al tutor acerca de las estrategias que faciliten su relación con las familias y el acercamiento de éstas al centro.

- Cuando no exista una relación positiva, o siempre que se considere necesario, se realizará una labor mediadora.

- Colaborar con el tutor en las actividades encaminadas a conseguir una mayor implicación de las familias en el proceso educativo de sus hijos a través de reuniones periódicas con los padres del grupo-clase, escuela de padres, entrevistas, mayor participación de las familias, etc.

\subsubsection{Colaborar en la atención a la diversidad}

Para conseguir avanzar en una adecuada atención a la diversidad, es fundamental que se tengan en cuenta dos exigencias básicas: la coordinación de todo el equipo educativo para determinar las líneas de actuación a seguir y la utilización de metodologías que primen la actividad del alumno sobre la del profesor para llegar al mayor número posible de alumnos. Esto contribuirá a la construcción de un currículum lo más flexible posible para tener que elaborar el mínimo de adaptaciones curriculares.

En lo referido a la atención a la diversidad, las actividades que puede llevar a cabo el trabajador social son las siguientes:

- Participar en el análisis de las necesidades educativas especiales y de las características del aula.

- Potenciar la coordinación de todo el equipo educativo, que actúa en un nivel o ciclo.

- Ayudar a un cambio en el papel del profesor: de emisor a dinamizador.

- Ayudar a un cambio en el papel del alumnado: de receptor a sujeto activo de su propio aprendizaje.

- Inducir a un cambio en las relaciones de los diversos subgrupos (profesor-profesor, alumno-alumno, profesor-alumno, escuela-familia, profesor-equipo de apoyo) con el objetivo de crear un clima en clase y en el centro que favorezca el tratamiento a la diversidad.

- Colaborar en la elaboración, seguimiento y evaluación de las adaptaciones curriculares. 
8.3. Realizar el estudio y la valoracion sociofamiliar de los casos detectados. Elaborar la propuesta de intervencion socioeducativa directa o canalizar hacia el recurso pertinente, con el posterior seguimiento y evaluación del caso.

La valoración sociofamiliar que se realice ha de atender a la globalidad del alumno, es decir, estudiar todos aquellos factores que le rodean y afecten como persona. Las valoraciones están insertas en un proceso, en un conjunto de actuaciones dirigidas a dar respuesta a las necesidades educativas y socio-familiares que rodean al alumno.

Las aportaciones del trabajador social a esta función se concretan en las siguientes actividades:

1. Estudio del entorno social y familiar del alumno empleando los instrumentos propios del trabajo social (entrevistas estructuradas/no estructuradas, cuestionarios, informes sociales, etc.) como parte de la evaluación diagnóstica.

2. Análisis y síntesis de la información recogida sobre las características del ambiente sociofamiliar del alumno, su entorno comunitario y su historial educativo.

3. Diseño de la intervención que consistirá en realizar un trabajo directo con la familia o se canalizará hacia el recurso pertinente.

4. Elaboración de informes técnicos cuando se requiera y sea necesario motivados por: cambios de escolarización, de centro ordinario a centro específico y viceversa; cambio del alumno a un grupo distinto de enseñanza-aprendizaje; gestión de determinados recursos (becas de comedor, de libros o de transporte, ayudas técnicas o instrumentales, etc.); a petición de otros organismos o servicios que intervengan en el caso; etc.

5. Colaborar en la elaboración, seguimiento y evaluación de las adaptaciones curriculares, incidiendo especialmente en los apartados de autonomía personal y socialización.

8.4. Participar en la elaboración y realización de actividades de orientación educativa y sociolaboral.

Cabe entender por orientación, en sentido amplio, la ayuda sistemática ofrecida a una persona para que llegue a un mejor conocimiento de sus características y potencialidades, a la aceptación de su propia realidad y al logro de su capacidad de autodirigirse. Todo ello, enfocado al desarrollo integral de su personalidad y a una contribución eficaz a la sociedad en que vive.

La orientación, si bien es para todos los sujetos, debe prestar particular atención a aquellos que presentan problemas de integración o 
adaptación al sistema educativo ordinario o a los recursos normalizados. Debe atender a todas las facetas de la personalidad del sujeto e intentar un desarrollo armónico y equilibrado de las mismas. Ha de basarse en las diferencias inter e intraindividuales. Ha de ser cooperativa, comprometiendo no sólo a los expertos, sino también al propio sujeto, a la familia, al profesorado y dirección del centro. Debe utilizar los recursos de la comunidad y coordinarlos con los de la escuela y la familia.

El trabajador social colaborará con el equipo interdisciplinar en la elaboración del programa de orientación educativa y sociolaboral que se oferta a los centros. Los programas de orientación sociolaboral que tradicionalmente se llevan a cabo en los centros escolares dan respuesta a los alumnos sobre la oferta educativa ordinaria, sin ofrecer demasiadas alternativas para aquellos alumnos que, por sus características personales, sociales, educativas, etc., no pueden acogerse a esta oferta. Corresponde al trabajador social llevar a cabo una serie de actividades para potenciar la orientación a todos y cada uno de estos alumnos, en especial a los alumnos con necesidades educativas especiales.

Las aportaciones del trabajador social a esta función se concretan en las siguientes actividades:

1. Estudio y valoración de aquellas condiciones sociofamiliares que influyan en la evolución y la continuidad del proceso de enseñanza-aprendizaje del alumno y su incorporación a la comunidad y al mundo laboral.

2. Participar junto con el psicopedagogo y los tutores en el diseño, ejecución y puesta en marcha del programa de orientación escolar, profesional y vocacional del centro.

3. Coordinación con otros Servicios e Instituciones hacia los que se puedan dirigir alumnos/as al acabar la escolaridad obligatoria que no puedan acogerse a la oferta educativa ordinaria.

4. Participar en la evaluación del Programa de orientación educativa y sociolaboral, y llevar a cabo un seguimiento para valorar la idoneidad del recurso propuesto al alumno.

\subsection{Colaborar con los distintos órganos de representación del centro escolar, según las necesidades.}

Como trabajadores sociales, nuestra intervención en los diferentes órganos de participación, relacionados con la escuela, vendrá dada, por un lado, por la demanda expresa del propio órgano y, por otro, por nuestra propia demanda de participación, derivada del estudio del centro, características y necesidades.

Nuestro trabajo se podrá realizar en los diferentes órganos con las siguientes actividades: 


\section{Equipo Directivo.}

- Informar al equipo directivo sobre aquellos aspectos relevantes relacionados con la comunidad educativa: interrelaciones entre sus diversos miembros, problemáticas que surgen, experiencias innovadoras y de interés para la comunidad, etc.

- Asesorar al equipo directivo en todas aquellas situaciones que surjan en el centro cuando sea necesario: actuación con determinados alumnos o familias cuya problemática sobrepasa al tutor, actuación en ámbitos más generales como puede ser la elaboración de un plan de prevención o actuación sobre la disciplina, etc.

- Información sobre recursos de la comunidad, legislación de interés, ayudas, etc.

2. Comisión de Coordinación Pedagógica

- Participar en dicha comisión en todos los temas que sean de su competencia: temas transversales, análisis del contexto, atención a la diversidad, acción tutorial, etc.

3. Claustro.

- Aportar aquellas informaciones generales y específicas sobre el entorno sociofamiliar del alumnado que sean relevantes para mejorar su comprensión de la situación y la atención al alumnado.

- Colaborar con el Claustro en las propuestas de programas para el centro y en la elaboración de estrategias generales de actuación a nivel del centro: programas de educación para la salud, de educación para la paz, escuelas de padres, desarrollo del Plan de Acción Tutorial, etc.

4. Consejo Escolar.

- Asesorar y orientar para la toma de decisiones en aquellas funciones que son propias del Consejo Escolar: admisión de alumnado, gestión de becas, programas concretos de actuación, reglamento de régimen interno, instrucciones de expedientes disciplinarios, etc.

5. Asociaciones de Padres de Alumnos (A.P.A.S.)

- Haremos referencia a las posibilidades de intervención con las A.P.A.S cuando hablemos de la función específica de asesoramiento a las familias.

6. Asociaciones de alumnos, Cámaras de delegados o Consejos de alumnos.

Nuestra participación en estos órganos estará encaminada a :

- Promover y estimular la constitución de estas asociaciones y consejos, ya que estos facilitan la representatividad y participación del alumnado en la vida del centro, favoreciendo también el aprendizaje de las formas democráticas de convivencia. 
- Asesorar en el proceso de elección de delegados y cargos.

- Participar, como asesores del alumnado, en la puesta en marcha de los mismos y en sus posteriores planes de actividad.

8.6. Asesorar a las familias y participar, en su caso, en el desarrollo de programas formativos de padres y madres de alumnos.

Los padres pueden proporcionar información relevante sobre sus hijos que sirva para dar contenido y sentido al trabajo que con ellos se desarrolla en el centro escolar. Del mismo modo, los profesores pueden colaborar con los padres en la búsqueda de respuestas a las necesidades que presenten los hijos en su desarrollo. Planteada de este modo, la participación de los padres en la escuela constituye un aspecto más e ineludible de su función educativa.

Desde el punto de vista legal, los padres tienen derecho constitucional a intervenir en la gestión y control de los centros escolares sostenidos con fondos públicos. Ahora bien, para que este reconocimiento legal se traduzca en acciones concretas y eficaces, es preciso que los padres conozcan y participen activamente en dichos cauces y creen canales propios de comunicación en el convencimiento de que sus puntos de vista y sus valores enriquecen extraordinariamente la vida de la escuela.

Las acciones para desarrollar la función de asesoramiento a las familias y a la Asociación de Padres de Alumnos, en su caso, pueden tener un enfoque individual o colectivo, según los objetivos específicos que se pretendan.

a) A nivel individual

1. Orientación y trabajo con las familias para procurar modificar las causas de origen familiar que puedan generar problemas de desarrollo personal, adaptación escolar y/o social en los alumnos.

2. Desarrollar programas de intervención familiar referidos a aspectos como autonomía personal, hábitos básicos, relaciones familiares, relaciones familia-escuela, etc.

3. Apoyo, atención y orientación a las familias de alumnos con necesidades educativas especiales.

4. Elaboración de programas de intervención educativa familiar para llevar a cabo las Adaptaciones Curriculares Individualizadas (ACIs).

b) A nivel colectivo

1. Promover la constitución de seminarios operativos de padres, profesores y/o alumnos, en los que se trabajen conjuntamente diversos aspectos que se desee potenciar o eliminar en la comu- 
nidad educativa, aspectos concretos sobre la participación en la gestión del centro, etc.

2. Colaboración con los tutores en la preparación y desarrollo de las reuniones que a lo largo del curso realizan con las familias de su grupo de alumnos.

3. Facilitar la formación de grupos operativos de padres y profesores que traten los diferentes problemas que pueden afectarles: problemas de disciplina, relacionales, interétnicos, etc.

4. Asesoramiento a las Asociaciones o Federaciones de Padres de Alumnos:

- Asesorar en la puesta en marcha y en el funcionamiento de la Asociación, proponiendo ideas y orientándoles en su acción.

- Si fuese necesario, servir de enlace entre el APA y otros órganos de gestión y representación del centro.

- Fomentar la participación de los padres en todo tipo de programas y acciones que se estén llevando a cabo en el centro.

- Participar en las reuniones de la Junta Directiva y asambleas del A.P.A., cuando se nos solicite.

- Aportarles datos para el conocimiento de la escuela y la comunidad educativa utilizando el análisis del contexto realizado desde el Proyecto Educativo del centro u otros estudios elaborados anteriormente.

- Colaborar en campañas de sensibilización organizadas por APAS o Federaciones de APAS dirigidas a promover actitudes positivas hacia la integración escolar y/o sociolaboral de los alumnos con necesidades educativas especiales, de minorías étnicas, actividades para fomentar la participación de padres, etc.

- Participar en la organización de actividades culturales y formativas, tanto para los alumnos como para las familias: semana cultural, actividades extraescolares, ciclos de charlas y conferencias, encuentros padres-profesores, jornadas de puertas abiertas, etc.

5. Promoción y participación de actividades formativas dirigidas a familias a través de escuelas de padres, charlas, conferencias y otro tipo de actividades.

El trabajador social podrá participar como colaborador en la organización de las actividades, intentando responder a las necesidades planteadas por los padres y a las detectadas a través del análisis del contexto medioambiental, el organizativo-institucional y las interacciones personales. También podrá asesorar en el diseño del programa de formación dirigido a los padres en todas sus fases. 
8.7. Elaborar y difundir materiales e instrumentos propios de la intervención socioeducativa.

El trabajador social elaborará documentos y materiales propios del Trabajo Social en el ámbito socioeducativo y /o adoptará instrumentos y materiales utilizados en la intervención social en otros campos adecuándolos a la especificidad de este ámbito.

1. Recopilar materiales e instrumentos existentes en la actualidad referidos a la intervención del trabajador social en el campo educativo.

2. Elaborar materiales específicos para programas o actividades concretas, tales como cuestionario de detección, catálogo de recursos socioeducativos, guía de alternativas al acabar la escolaridad, materiales para el asesoramiento y la formación de padres, etc.

3. Elaborar y divulgar materiales informativos para padres, profesores y alumnos sobre recursos socioeducativos del entorno.

4. Proporcionar a los profesores materiales de apoyo para su acción tutorial (cuestionarios de detección de indicadores de riesgo social, cuestionario de detección de alumnos con necesidades educativas especiales, etc.).

5. Contribuir a la renovación de enfoques y técnicas de innovación educativa e intervención sociofamiliar.

6. Realizar investigaciones sobre cuestiones que son objeto de su intervención profesional, así como sobre distintos aspectos de la comunidad educativa con la que trabaja, que sirvan, al mismo tiempo, para contribuir al desarrollo teórico-práctico del Trabajo Social en este campo.

\section{BIBLIOGRAFÍA}

ÁLVAREZ, M. y FERNÁNDEZ, R. (1989): Programa de orientación de estudios y vocacional al término de la escolaridad obligatoria. Promociones y Publicaciones Universitarias, S.A. Barcelona.

CAMPO, M.² A. y LASMARIAS, M. (1993): El trabajador social en los equipos interdisciplinares. Programa de intervención en el contexto sociofamiliar. Documento de Orientación Educativa e Intervención Psicopedagógica (M. ${ }^{\circ}$ de Educación y Ciencia).

DECRETO 136/1984, sobre creación y funcionamiento de los Servicios Psicopedagógicos Escolares.

DECRETO 13/1994, por el que se regulan los Servicios Especializados de Orientación Educativa, Psicopedagógica y Profesional ( DOGV n ${ }^{\circ} 2.320$, de 28-7-94). 
ESTRADA LORCA, M. y MOLINA SÁNCHEZ, M." V. (1991): "La interdisciplinariedad en la orientación educativa: respuesta a la diversidad" en Respuesta educativa ante la diversidad. Amarú Ediciones. Salamanca.

HERRANZ HERRANZ, C. (1991): "La investigación-acción como modelo de intervención psicopedagógica" en Respuesta educativa ante la diversidad. Amarú Ediciones. Salamanca.

IFAPLAN (1988): Nuevos temas y lugares educativos. Editorial Popular. Ministerio de Educación y Ciencia. Madrid.

JIMÉNEZ PUADO, C. (1990): Trabajo Social en el sistema educativo. Revista "Documentación Social", n79, pp. 145-159. Madrid. Cáritas Española.

LEY ORGÁNICA 1/1990, de Ordenación General del Sistema Educativo (LOGSE) ( BOE,4-11-90).

MINISTERIO DE EDUCACIÓN Y CIENCIA (1989): Libro Blanco para la Reforma del Sistema Educativo. Madrid.

ORDEN 10 de Marzo de 1995, por la que se determinan las funciones de cada profesional dentro de estos equipos.

PÉREZ SERRANO, G. (1990): Investigación-acción: aplicaciones al campo social y educativo. Dykinson. Madrid.

ROSELLÓ NADAL, E. y SAAVEDRA MUÑOZ, M. (1995): La intervención del trabajador social en los Servicios Psicopedagógicos Escolares. Conselleria de Educación y Ciencia. Generalitat Valenciana. 